ARTICLE

Received 21 Jul 2014 | Accepted 11 Mar 2015 | Published 21 Apr 2015

DOI: $10.1038 /$ ncomms7899 OPEN

\title{
A STIM2 splice variant negatively regulates store-operated calcium entry
}

\author{
Anna-Maria Miederer ${ }^{1, \star}$, Dalia Alansary ${ }^{1, \star}$, Gertrud Schwär ${ }^{1,2}$, Po-Hsien Lee ${ }^{3}$, Martin Jung ${ }^{4}$, \\ Volkhard Helms ${ }^{3} \&$ Barbara A. Niemeyer ${ }^{1}$
}

Cellular homeostasis relies upon precise regulation of $\mathrm{Ca}^{2+}$ concentration. Stromal interaction molecule (STIM) proteins regulate store-operated calcium entry (SOCE) by sensing $\mathrm{Ca}^{2+}$ concentration in the ER and forming oligomers to trigger $\mathrm{Ca}^{2+}$ entry through plasma membrane-localized Orai1 channels. Here we characterize a STIM2 splice variant, STIM2.1, which retains an additional exon within the region encoding the channel-activating domain. Expression of STIM2.1 is ubiquitous but its abundance relative to the more common STIM2.2 variant is dependent upon cell type and highest in naive T cells. STIM2.1 knockdown increases SOCE in naive CD4 ${ }^{+}$T cells, whereas knockdown of STIM2.2 decreases SOCE. Conversely, overexpression of STIM2.1, but not STIM2.2, decreases SOCE, indicating its inhibitory role. STIM2.1 interaction with Orai1 is impaired and prevents Orai1 activation, but STIM2.1 shows increased affinity towards calmodulin. Our results imply STIM2.1 as an additional player tuning Orai1 activation in vivo.

\footnotetext{
${ }^{1}$ Molecular Biophysics, Center for Integrative Physiology and Molecular Medicine (CIPMM), School of Medicine, Saarland University, Building 48, Homburg 66421, Germany. ${ }^{2}$ Department of Biophysics, Center for Integrative Physiology and Molecular Medicine (CIPMM), School of Medicine, Saarland University, Homburg 66421, Germany. ${ }^{3}$ Center for Bioinformatics, Saarland University, Campus E2 1, R. 315, PO Box 151150, Saarbrücken 66041, Germany. ${ }^{4}$ Department of Medical Biochemistry and Molecular Biology, Saarland University, Building 44, Homburg 66421, Germany. * These authors contributed equally to this work. Correspondence and requests for materials should be addressed to B.A.N. (email: barbara.niemeyer@uks.eu).
} 
A mplitude, duration and kinetics of $\mathrm{Ca}^{2+}$ signals code for differential changes in gene expression, degranulation, cytokine release, proliferation and migration. These diverse functions require tight control and regulation of $\mathrm{Ca}^{2+}$ influx. In immune cells, the major influx pathway for $\mathrm{Ca}^{2+}$ is encoded by components of the store-operated calcium entry (SOCE) machinery. Here, CRAC $\left(\mathrm{Ca}^{2+}\right.$ release-activated $\left.\mathrm{Ca}^{2+}\right)$ channels encoded by the Orai genes (Orail-3) are activated by endoplasmatic reticulum (ER)-localized stromal interaction molecule (STIM) (STIM1 and STIM2) proteins when the $\mathrm{Ca}^{2+}$ concentration of the ER $\left(\sim 400-600 \mu \mathrm{M}^{1}\right)$ is decreased ${ }^{2}$. In contrast to STIM1, the major activator of Orail upon effector T-cell activation, the functional role of STIM2 is less well understood. The first studies on STIM2 in 2006 described an inhibitory effect of STIM2 on STIM1-mediated SOCE, as well as its constitutive and store-independent activation of Orail (ref. 3). An short interfering RNA (siRNA)-based screen for regulators of basal cytosolic $\mathrm{Ca}^{2+}$ identified STIM2, calmodulin (CaM) and the plasma membrane $\mathrm{Ca}^{2+}$ ATPase (PMCA) as critical components with downregulation of STIM2 decreasing basal $\mathrm{Ca}^{2+}$ and downregulation of CaM or plasma membrane $\mathrm{Ca}^{2+}$ ATPase increasing basal $\left[\mathrm{Ca}^{2+}\right]$ (ref. 4). The authors also showed that STIM2 responds to smaller decreases in ER $\left[\mathrm{Ca}^{2+}\right]$ with an EC50 of $406 \mu \mathrm{M}$ compared with $210 \mu \mathrm{M}$ for STIM1 (see also ref. 5). In addition to the decreased affinity of the $\mathrm{Ca}^{2+}$-binding EF hand, the STIM2 SAM domain displays an increased conformational stability when compared with STIM1 (ref. 5). In contrast to STIM1, the SAM domain of STIM2 contains only a single $\mathrm{N}$-glycosylation site. Modifications of the glycosylation sites within the SAM domain of STIM1 lead to altered oligomerization rates and current size as well described by a reaction diffusion model $^{6}$.

Whether the effect of STIM2 on SOCE is activation or inhibition is controversial, and a recent published computational model of regulation of basal $\mathrm{Ca}^{2}+$ homeostasis did not consider a role of STIM2 (ref. 7). A second confounding factor in the analysis of STIM2 function is an existing uncertainty regarding its translational start site. In the original report describing cloning of STIM2, Williams et al. ${ }^{8}$ postulated translation from a non-AUG codon around L88, a view that was later revised by Graham et al., who showed that STIM2 contains an unconventionally long signal peptide with translation from a conserved Met, which can cause incomplete protein translocation into the ER and leads to a small amount of cytosolic STIM2, responsible for pre-coupled and non-store-operated activation of STIM2-Orail complexes 9 . In addition to its role in maintaining basal $\mathrm{Ca}^{2+}$ and controlling ER $\mathrm{Ca}^{2}+$ levels ${ }^{4}$, STIM2 activates Orail signalling upon submaximal store depletion, driving $\mathrm{Ca}^{2+}$ oscillations due to partially pre-coupled STIM2-Orail population ${ }^{10-14}$. STIM2 and Orail form a $\mathrm{Ca}^{2+}$-sensitive and thapsigargin-insensitive complex in cortical neurons ${ }^{15}$, with loss of STIM2-protecting neurons from store-mediated hypoxic neuronal death ${ }^{16}$. In cancer, controversial roles for STIM2 as both a tumour suppressor or as a potential oncogene have been postulated ( ${ }^{17-}$ 20 , reviewed in ref. 14). Recent publications also present growing evidence for a key role for STIM2 in tumour immunity by $\mathrm{CD} 8^{+}$ cells $^{21}$ and in several immune diseases. In a murine multiple sclerosis model, STIM2-deficient mice were shown to be less susceptible to autoimmune encephalomyelitis (EAE), suggesting a role for STIM2-mediated $\mathrm{Ca}^{2}+$ signalling in autoimmune disease $^{22,23}$. In contrast to the EAE model, deficient STIM2 signalling caused salivary gland autoimmune pathology in Primary Sjögren's Syndrome ${ }^{24}$.

Here we report the identification and detailed characterization of a novel STIM2 splice variant, named STIM2.1, which acts as an inhibitory regulator of STIM-mediated activation of Orai channels. The additional exon of STIM2.1 is spliced into the channel-activating domain (CAD), immediately upstream of the sequences essential for binding of Orail (refs 13,25), disabling STIM2.1 CAD from activating Orail and altering the CAD domain affinity for CaM binding.

\section{Results}

Identification of a novel STIM2 splice variant. We discovered the existence of two additional STIM2 splice variants by database mining, namely STIM2.1 containing an additional exon 9 and STIM2.3 containing an alternative exon $13\left(13^{*}\right)$ leading to an upstream end of translation and a transcript shortened by $444 \mathrm{bp}$ $(\sim 17 \mathrm{kDa})$ (Fig. 1a). All reports on STIM2 are conducted with the STIM2.2 variant. Although we were unable to detect messenger RNA (mRNA) expression of STIM2.3 in lymphocytes by different PCR-based strategies, we identified STIM2.1 in a conventional PCR reaction with primers (for, rev) flanking exon 9. Figure $1 \mathrm{~b}$ shows two different PCR products in human $\mathrm{CD} 8{ }^{+} \mathrm{T}$ cells as well as in Jurkat $\mathrm{T}$ cells and primary monocytes. Exon 9-specific quantitative reverse transcription (RT)-PCR primers were derived (Fig. 1a; Supplementary Table 1), PCR products were confirmed by DNA sequencing and relative expression levels of STIM2.1 (with exon 9, NM001169118) and of STIM2.2 (without exon 9, NM020860) were tested using template complementary DNA (cDNA) of naive and stimulated $\mathrm{CD}^{+}{ }^{+} \mathrm{T}$ cells from at least three different primary human blood donors. As also indicated by conventional PCR (inset in Fig. 1c), STIM2.1 expression is highest in naive $\mathrm{T}$ cells but is reduced upon stimulation with anti-CD3/ anti-CD28-coated beads. Seven hours after bead contact, the ratio of STIM2.2/STIM2.1 transiently increases to $4 \pm 0.5$, but decreases again to a ratio of $1.6 \pm 0.56$ after $72 \mathrm{~h}$ following initial bead contact (Fig. 1c). A reduction of mRNA expression is also seen for STIM2.2 and for STIM1 although STIM1 expression recovers after $72 \mathrm{~h}$, whereas total STIM2 mRNA and STIM2 protein remains reduced in stimulated cells (Fig. 1c; Supplementary Fig. 1a,b). We proceeded to test splice-specific expression in a number of cell lines and tissues and plotted the ratio of STIM2.2 expression over STIM2.1 expression (Fig. 1d). Highest expression of the novel STIM2.1 (lowest ratio) is detected in naive $\mathrm{CD} 4^{+}$and $\mathrm{CD} 8{ }^{+}$ T cells. cDNA from glioblastoma samples (12 patients) showed the highest expression of STIM2.2 with little STIM2.1. In summary, we could detect expression of STIM2.1 in all tested human cell lines and primary cells, although expression was always lower than STIM2.2. Alignment of the exon 9 amino acids (VASSYLIQ) shows a high degree of conservation between different species. We also tested for STIM2.1 (VAASYLLQ) expression in lymphocytes from Mus musculus and were able to detect two bands by conventional PCR (Supplementary Fig. 1d).

Knockdown of STIM2.1 alters SOCE in primary cells. Because naive $\mathrm{CD}^{+}{ }^{+}$and $\mathrm{CD} 8{ }^{+} \mathrm{T}$ cells showed the highest absolute expression of STIM2.1 ( $2-\Delta$ Cq: $0.68 \pm 0.35)$, with an average ratio of STIM2.2/STIM2.1 expression of $1.6 \pm 0.4$ (5 donors) for naive $\mathrm{CD}^{+}{ }^{+}$cells and a ratio of $1.5 \pm 0.14$ (3 donors) for $\mathrm{CD} 8{ }^{+}$cells (see Fig. 1c), these cells lent themselves for investigating endogenous STIM2.1 function. Although limited by the very short sequence of exon 9, we devised splice-specific siRNA targeting either exon 9 or the exon 8/exon 10 boundary (Supplementary Table 1). Efficiency of knockdown was tested by qRT-PCR 14-18 h after siRNA transfections on two consecutive days. STIM2.1-specific siRNA decreased cDNA expression to an average of $44 \pm 6 \%$ of its non-silenced control without exerting a significant effect on the expression of STIM2.2 or on STIM1 (Fig. 2a), neither did the siRNA show off-target or indirect effects on the expression of Orail (97\% of control). Given an mRNA 
a
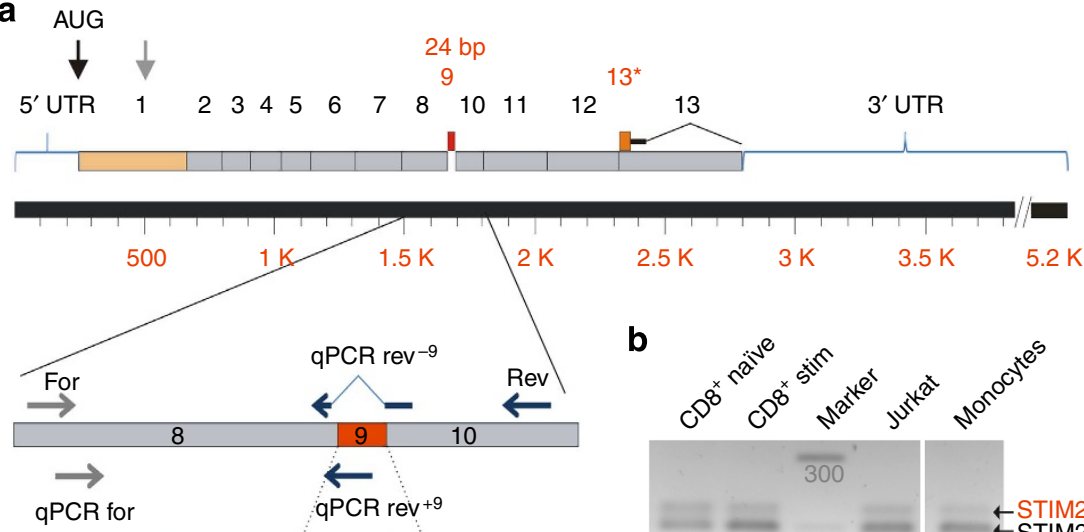

AEMQLAIAKDE VAASYLIQ AEKIKKKRS
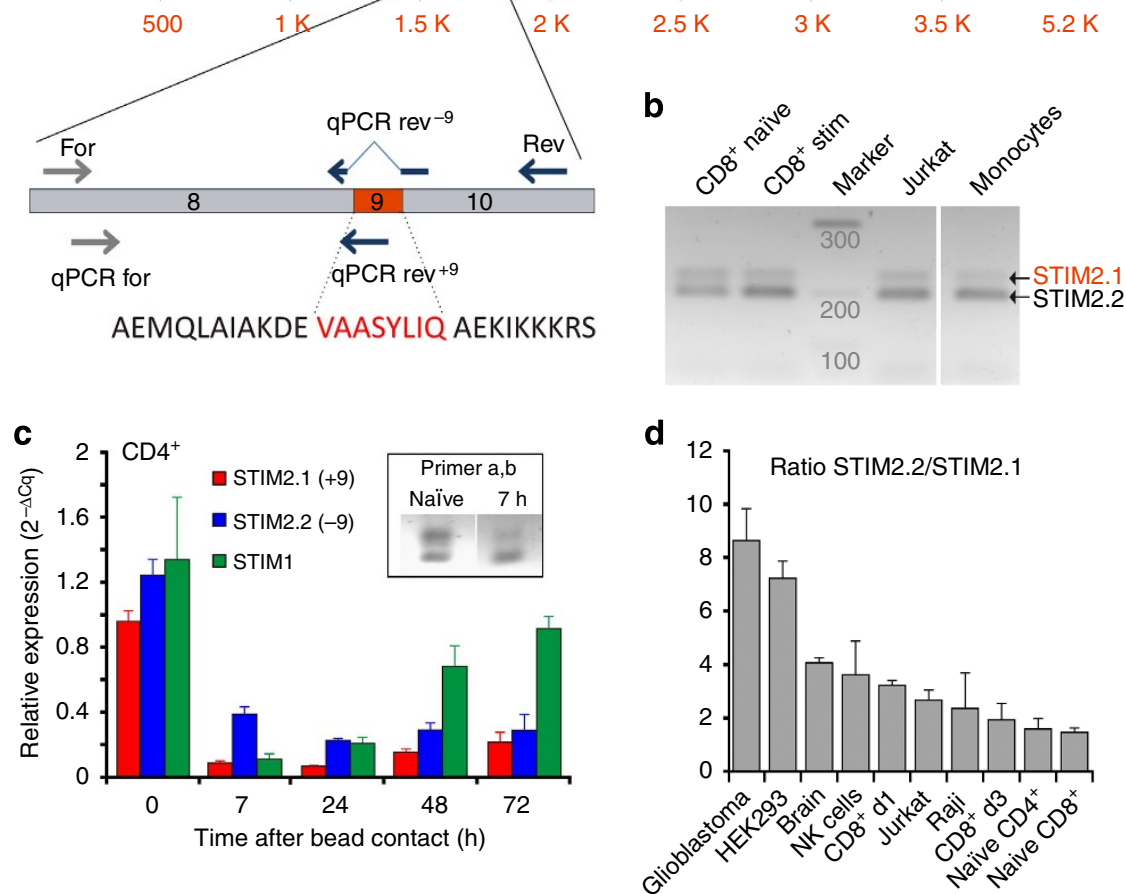

Figure 1 | Identification of a novel STIM2 splice variant. (a) Schematic representation of human STIM2 mRNA with exon boundaries. Highlighted in red are exon 9 and $13^{\star}$ present only in the splice variants STIM2.1 and STIM2.3, respectively. The enlarged region shows the primer pairs used in conventional (for: grey arrow and rev: blue arrow) or quantitative ( $9 P C R$ for, GPCR rev -9 , QPCR rev +9 ) PCRs to detect and analyse splice variant expression. (b) Image showing PCR amplification products obtained with primer pair (for and rev) using CDNA from naive and stimulated human CD8 ${ }^{+}$T cells, Jurkat T cells and human monocytes. (c) Relative expression of STIM1, STIM2.1 and STIM2.2 in naive and stimulated CD4 ${ }^{+}{ }^{\text {T cells with indicated time periods after }}$ initial contact with anti-CD3/anti-CD28-coated beads. Expression was normalized to that of TBP (three donors) (d) Ratio of STIM2.2/STIM2.1 expression obtained by qRT-PCR using reverse-transcribed mRNA isolated from the cell types indicated below the bars (3-12 donors or independent RNA preparations).

expression ratio of STIM2.2/STIM2.1 of 1.6 and a knockdown efficiency of $\sim 50 \%$, we expected a reduction of total STIM2 protein of about $20 \%$, which we indeed observed (Supplementary Fig. 1c). Splice-specific knockdown of STIM2.2 was not as successful, leading to reduction of STIM2.2 expression to an average of $68 \pm 8 \%$ and to small $(\sim 10 \%)$ off-target or indirect effects on STIM1, STIM2.1 (Fig. 2b) and Orail (91\% of control) expression. Measurements of $\left[\mathrm{Ca}^{2+}\right]_{i}$ of naive $\mathrm{T}$ cells showed that siRNA specific to STIM2.1 did not have a significant effect on basal $\left[\mathrm{Ca}^{2+}\right]_{\mathrm{i}}$; however, it led to a significant increase in rate, peak and plateau of SOCE (Fig. 2c-g). In contrast and despite the relatively weak downregulation of STIM2.2 expression, $\mathrm{Ca}^{2+}$ imaging of primary naive $\mathrm{CD}^{+}$cells revealed that STIM2.2 siRNA significantly decreased basal $\mathrm{Ca}^{2+}$ concentrations in bath solutions with different $\left[\mathrm{Ca}^{2+}\right]_{\mathrm{o}}$, (Fig. 2h,i), as expected upon downregulation of STIM2 (ref. 4), decreased the size of the thapsigargin (Tg)releasable pool, which is very small and variable in naive T cells (see Supplementary Fig. 1e) and significantly reduced SOCE (Fig. 2j-l), demonstrating that expression of STIM2.2 in naive cells is a significant determinant of their $\mathrm{Ca}^{2+}$ homeostasis, and confirming that STIM2.2 is a regulator not only of basal but also of storeoperated $\mathrm{Ca}^{2}+$ homeostasis. STIM2.1 siRNA treatment showed opposite effects, strongly indicating that STIM2.1 acts as a break or inhibitory regulator on STIM1/STIM2-mediated SOCE in vivo.
Upregulation of STIM2.1 decreases T-cell SOCE. If downregulation of STIM2.1 increases SOCE, we reasoned that upregulation of STIM2.1 in Jurkat T cells may decrease SOCE and therefore expressed either STIM2.2 or STIM2.1 after cloning exon 9 into a STIM2 expression vector with the full STIM2 signal peptide sequence and where the fluorescent mcherry-coding sequence replaced the variable domain NT 1966-2160 (I648K711), retaining $121 \mathrm{C}$-terminal aa residues (see also below). Figure 3a shows that overexpression of STIM2.2 in Jurkat T cells leads to an increased basal $\left[\mathrm{Ca}^{2+}\right]$, a reduced $\mathrm{Tg}$-releasable store content but also an increased rate, peak and plateau of SOCE (Fig. 3b-e). Overexpression of STIM2.1 showed similar effects to STIM2.2 on basal $\mathrm{Ca}^{2}+$ and Tg-releasable store content but, in contrast to STIM2.2, significantly decreased rate, peak and plateau of SOCE (Fig. 3c,e). These results complement the siRNA data concerning SOCE (Fig. 2) and confirm an opposite role for both splice variants in regulation of SOCE after full store depletion.

STIM2.1 is unable to activate Orai1. To further analyse the function of STIM2.1, we cloned exon 9 into an existing STIM2.2 expression vector, where the STIM1 signal peptide sequence is followed by YFP and the sequence of STIM2 starting at C102 


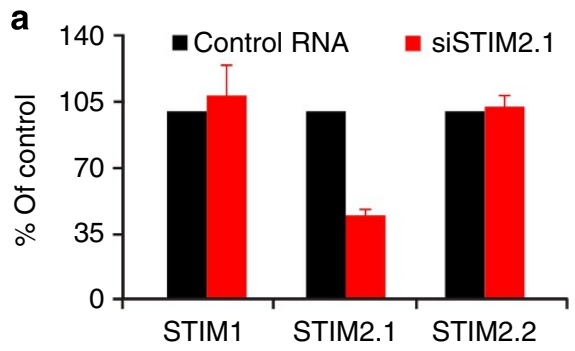

C

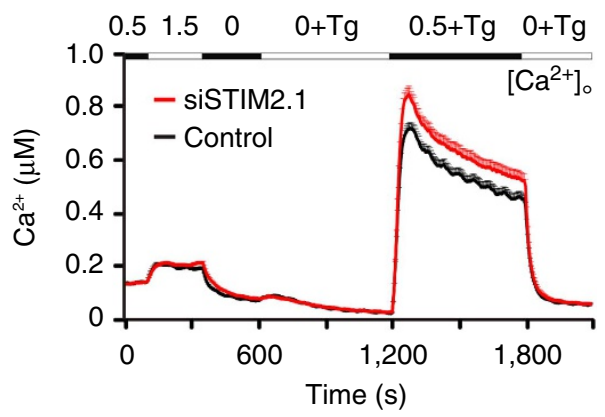

h

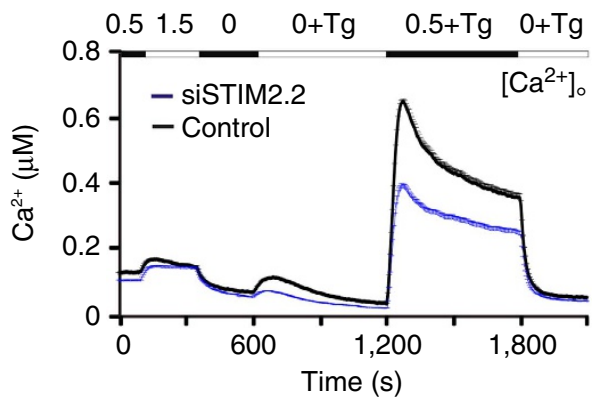

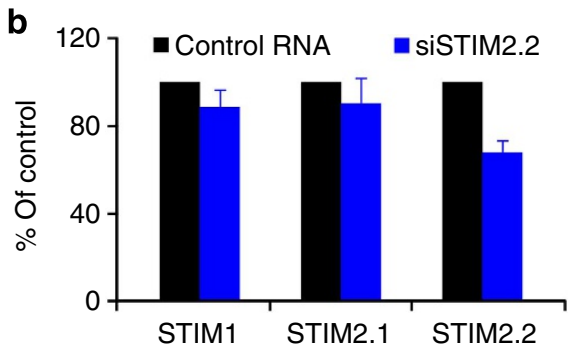

d
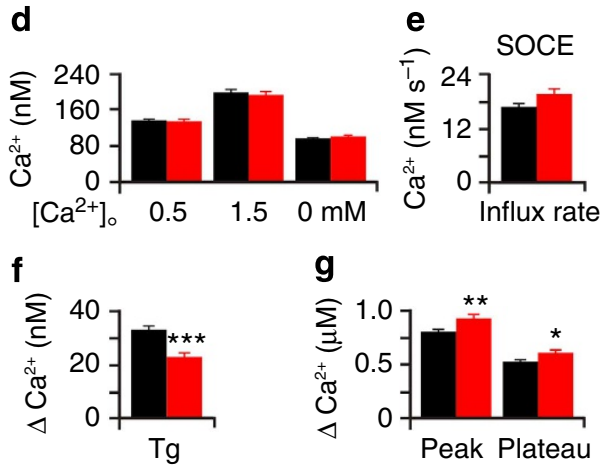

i

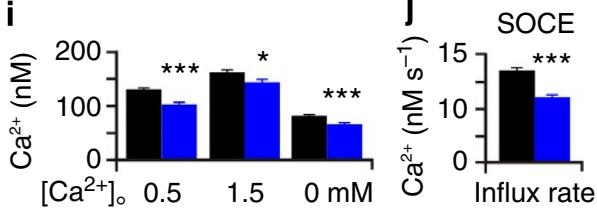

$\mathbf{k}$
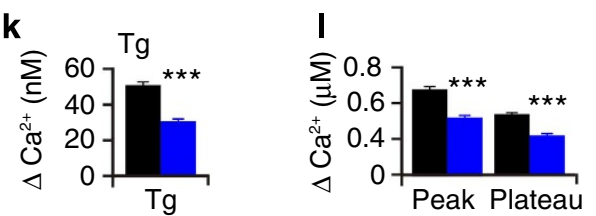

Figure 2 | Specific knockdown of STIM2.1 alters SOCE in primary cells. (a) Relative expression of STIM1, STIM2.1 and STIM2.2 after transfection with siRNA against exon 9 (STIM2.1) red or siRNA against exon 8/10 boundary (b, STIM2.2, blue), corresponding control transfection, black bars. (c) Traces showing average changes in intracellular $\mathrm{Ca}^{2+}$ concentration $\left[\mathrm{Ca}^{2+}\right]_{i}$ over time in $\mathrm{CD} 4{ }^{+}$T cells transfected with control (black, $\left.n=698\right)$ or siRNA against STIM2.1 (red, $n=579$ ) in response to perfusion of different $\left[\mathrm{Ca}^{2+}\right]_{0}$ indicated in the upper bar. $(\mathbf{d}-\mathbf{g})$ Quantification of changes in $\left[\mathrm{Ca}{ }^{2+}\right]_{i}$ measured in c. (h) Traces showing changes in $\left[\mathrm{Ca}^{2+}\right]_{\mathrm{i}}$ in $\mathrm{CD} 4{ }^{+}$T cells treated as in $\mathbf{c}$ after transfection with control (black, $n=978$ ) or siRNA against STIM2.2 (blue, $n=776$ ). (i-I) Quantification of changes in $\left[\mathrm{Ca}^{2+}\right]_{i}$ measured in $\mathrm{h}$. Measurements shown in $\mathbf{c}-\mathbf{I}$ are obtained from cells of three independent blood donors and $\geq 8$ experiments. ${ }^{\star} P<0.05,{ }^{\star \star} P<0.01,{ }^{\star \star \star} P<0.001$, Student's $T$-test. Data obtained are presented as mean \pm s.e.m.

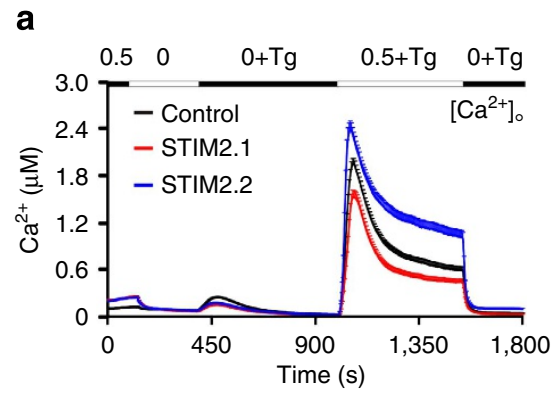

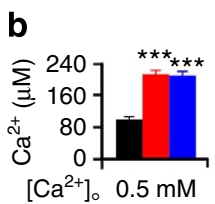

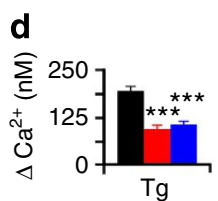

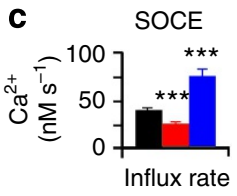

e

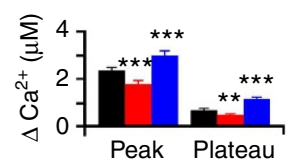

Figure 3 | Upregulation of STIM2.1 decreases T-cell SOCE. (a) Traces showing average changes in intracellular $\mathrm{Ca}^{2+}$ concentration $\left[\mathrm{Ca}{ }^{2+}\right]_{i}$ over time in Jurkat T cells transfected with control (black), STIM2.1-mCherry (red) or STIM2.2-mCherry (blue) in response to perfusion of different [Ca $\left.{ }^{2+}\right]_{0}$ indicated in the upper bar. (b-e) Quantification of changes in $\left[\mathrm{Ca}^{2+}\right]_{\mathrm{i}}$ measured in a from 131 to 216 cells of 6 experiments. ${ }^{\star \star} P<0.01,{ }^{\star \star \star} P<0.001$, Student's $T$-test. Data obtained are presented as mean \pm s.e.m.

(ref. 4) or into a vector containing the full STIM2 signal peptide (M1-G101) and coding sequence with no added fluorescent tag ${ }^{10}$. Expression of each of these constructs in HEK293 cells stably expressing Orail (HEKO1) cells revealed a significant increase in the basal $\mathrm{Ca}^{2}+$ content of the cytosol upon expression of STIM2.2, as well as a significant increase in SOCE when 
compared with an equimolar vector control. Figure 4a-e shows the results for the $\mathrm{N}$-terminally tagged constructs (see also ref. 4). Expression of STIM2.1 alone did not increase basal $\left[\mathrm{Ca}^{2+}\right]_{\mathrm{i}}$ nor significantly affected the Tg-releasable pool. However, in contrast to STIM2.2, STIM2.1 is not only unable to activate SOCE upon store depletion but also acts as an inhibitor of SOCE in the vector-only-transfected control (Fig. 4a-e). The YFP or mcherry fluorescence of the directly tagged constructs (Fig. 3) clearly indicated expression, which was also confirmed by western blot analysis (Supplementary Fig. 1f). To directly measure channel activity, we conducted whole-cell patch-clamp recordings of STIM2.1 and STIM2.2 expressed in HEKO1 cells with a pipette solution containing $\mathrm{IP}_{3}$ and BAPTA (1,2-bis(o-aminophenoxy) ethane- $N, N, N^{\prime}, N^{\prime}$-tetraacetic acid). As expected, STIM2.2 displayed inward currents that showed an initial rapid phase (store operated) and a second slower phase of increasing current density indicative of loss of a diffusive factor from the cytosol ${ }^{10}$ (Fig. 4f). Similar to the results shown in Fig. 4a, STIM2.1 was unable to sustain Orail currents (Fig. $4 \mathrm{f}-\mathrm{h}$ ). We also tested the ability of STIM2.1 and STIM2.2 to activate Orai2 channels and obtained a related result, namely a large increase in basal $\mathrm{Ca}^{2+}$ and Orai2-mediated SOCE can be observed with STIM2.2 but not with STIM2.1 (Supplementary Fig. 1g-k). The results shown in Fig. 4 indicate that STIM2.1 by itself either may be unable to cluster and localize to ER-PM (plasma membrane) junctional regions, unable to bind to and/or unable to gate Orail channels effectively. Thus, we set out to investigate the mechanisms underlying the observed functional differences between STIM2.1 and STIM2.2.

STIM2.1 interaction with Orail is impaired. We used total internal reflection fluorescence (TIRF) microscopy to assess the ability of STIM2.1 to oligomerize, translocate towards the ER-PM junctional regions, to recruit Orail to STIM2 punctae and to interact with Orail. HEK239 cells were co-transfected with Orai1-mEGFP and STIM2.1 or STIM2.2 tagged with mcherry replacing aa 648-711 (see Fig. 3) and treated with $1 \mu \mathrm{M} \mathrm{Tg}$ for $\sim 15$ min to induce store depletion followed by end-point analysis. Figure 5a shows that both STIM2 variants as well as Orai1 formed clusters. Manders coefficient (M1 and M2) analysis was performed on background-subtracted images of cells using the JACoP plugin in Fiji ${ }^{26}$. M1 shows the co-localization of STIM2.1 or STIM2.2 with Orail, whereas M2 depicts the fraction of Orai1 co-localizing with STIM2.1 or STIM2.2. We observed a small decrease in the fraction of Orail co-localizing with STIM2.1 (Fig. 5b, M2 red bar); however, STIM2.1 clearly is still able to colocalize with Orail (Fig. 5b, M1 red bar). We also did not observe any differences in mutual co-localization among STIM2 splice variants (Supplementary Fig. 2). In stark contrast, Förster resonance energy transfer (FRET) measurements yielded a very different result: as can be appreciated from the FRET channel images shown in Fig. 5a and quantified in Fig. 5c, STIM2.1 shows a marked defect in the apparent FRET efficiencies when compared with STIM2.2. These results explain the inability of STIM2.1 to activate Orail, but also indicate that either a second interaction site is unperturbed (leading to co-localization) or that an interaction still exists but that the distance between the fluorescent tags now is too far to measure FRET. Controls and correction factors are described in detail in the Methods section. As shown in Fig. 5d, both STIM2.2 and STIM2.1 formed extensive punctae also without stimulation, as predicted from the lower EF-hand $\mathrm{Ca}^{2+}$ affinity of STIM2, pointing towards normal luminal $\mathrm{Ca}^{2+}$ sensing and oligomerization mechanisms within STIM2.1. The fact that most of these clusters do not contain Orail indicates that either STIM2 is not yet completely unfolded or possibly that additional cytosolic factors prevent extensive Orail co-clustering. Nevertheless, the high resting calcium (Fig. 4) with overexpression of STIM2.2 indicates the presence of clusters with functional coupling between Orail and STIM2.2.

STIM2.1 opposes STIM2 and STIM1 function. Given the results shown in Figs 2 and 3, we also investigated heterologous co-
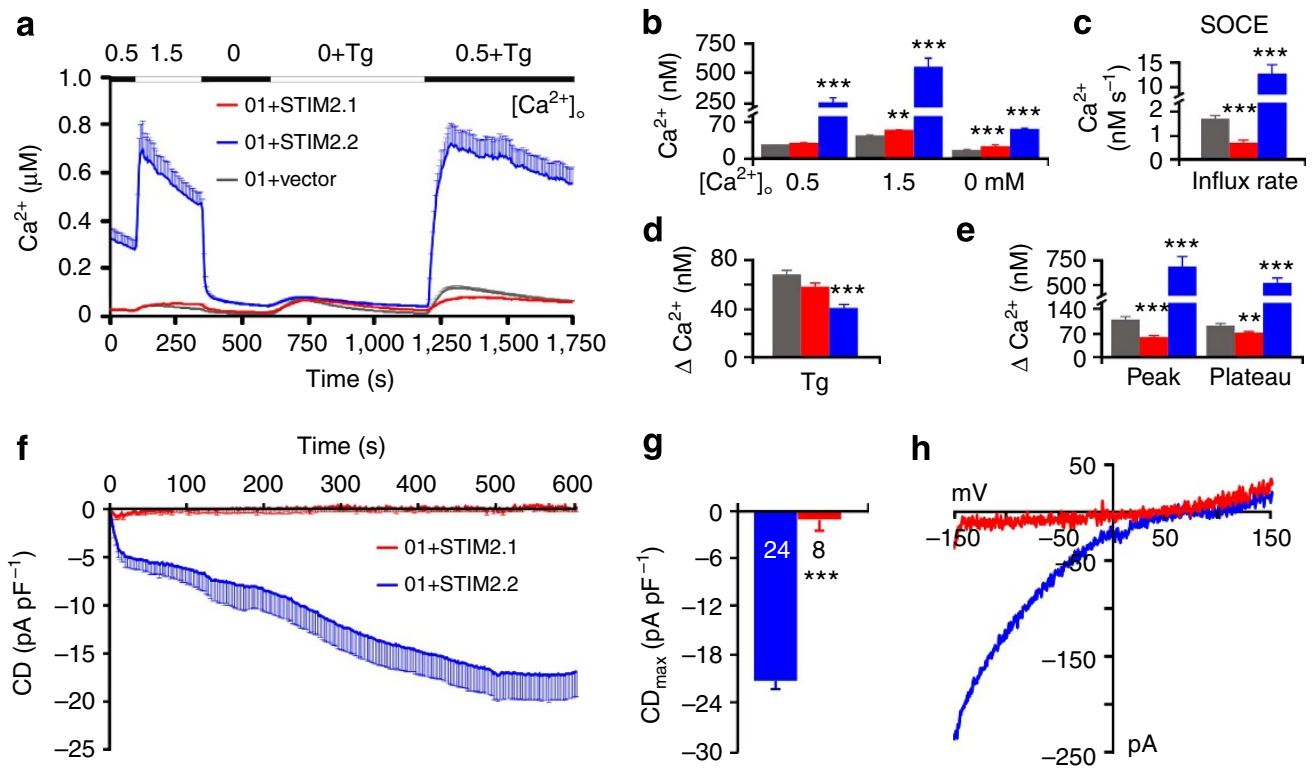

Figure 4 | STIM2.1 is unable to activate Orai1 in overexpression systems. (a) Traces showing average changes in intracellular Ca ${ }^{2+}$ concentration $\left[\mathrm{Ca}^{2+}\right]_{i}$ over time in response to perfusion of different $\left[\mathrm{Ca}^{2+}\right]_{0}$ indicated in the upper bar in HEKO1 cells transfected with YFP-STIM2.1 (red, $\left.n=96\right)$ or YFP-STIM2.2 (blue, $n=107$ ) or an empty vector control (grey, $n=72$ ), 6 experiments per condition. (b-e) Quantification of changes in $\left[C a^{2}+\right]_{i}$ measured in a. (f) Average traces showing whole-cell current density (CD) over time extracted at $-130 \mathrm{mV}$ in HEKO1 cells transfected with YFP-STIM2.1 (red) or YFP-STIM2.2 (blue). (g) Average maximum CD recorded from cells measured in $\mathbf{f}$. (h) Current-voltage (I- $V$ ) relationship of representative cells recorded in f. ${ }^{\star \star} P<0.01,{ }^{\star \star \star} P<0.001$, Student's $T$-test. Data obtained are presented as mean \pm s.e.m. 
a
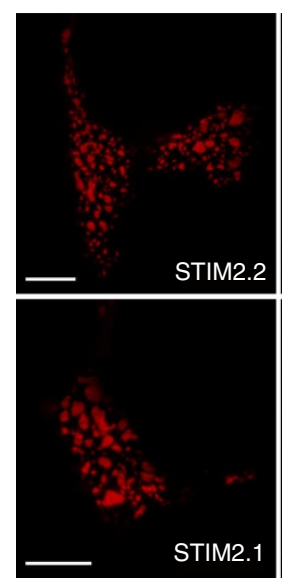

After thapsigargin
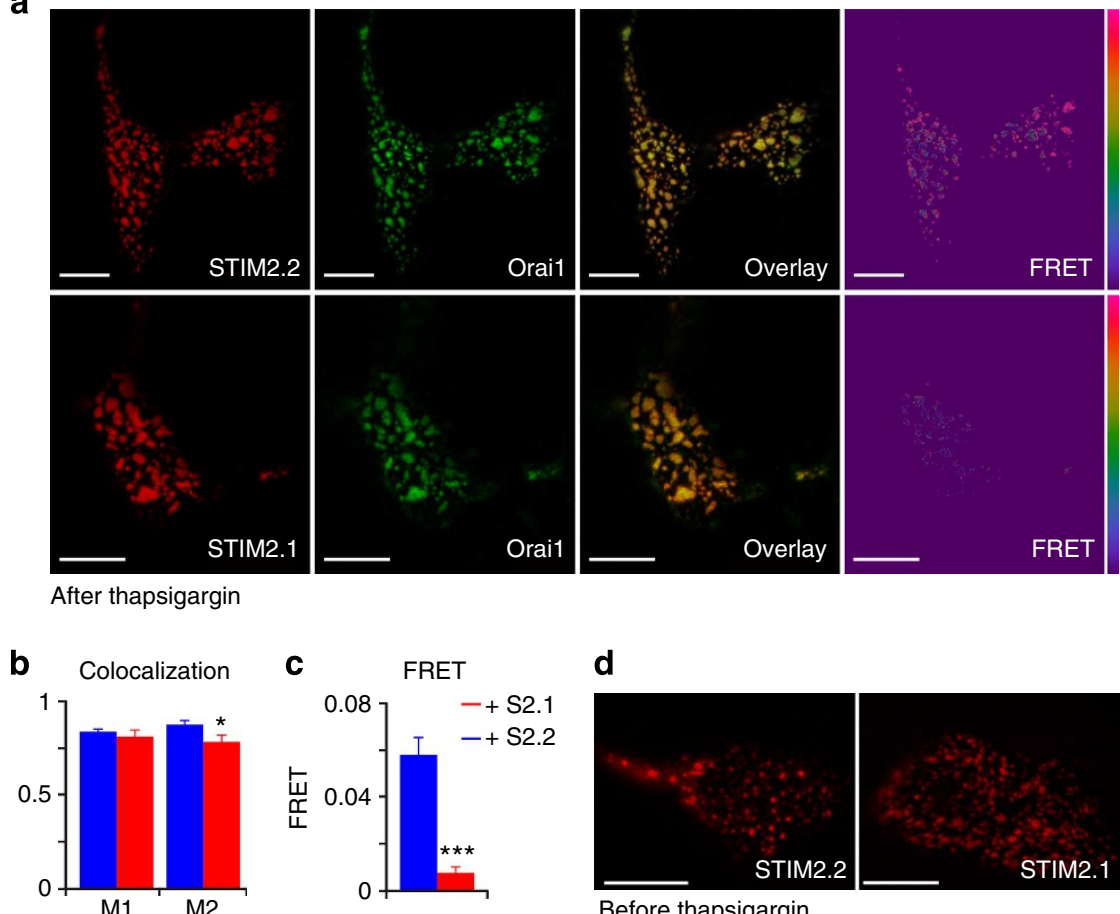

Figure 5 | Co-localization and interaction analyses. (a) Images showing HEKWT cells expressing Orai1-GFP (green), STIM2.1- (upper panel) or STIM2.2 (lower panel)-mCherry (red) and the corresponding overlay and FRET images (LUT 0-0.3). (b) Analysis of co-localization of STIM2 and Orai1 using Manders coefficient (M1) or vice versa (M2) ( $n=9$ images for both conditions) in the same cells as analysed for FRET (STIM2.1-Orai1 $n=36$; STIM2.2Orai1 $n=31$, from 3 independent experiments). (c) Quantification of the FRET signals measured in $\mathbf{a}$. ${ }^{\star} P<0.05$, ${ }^{\star \star \star} P<0.001$, Student $T$-test. Data obtained are presented as mean \pm s.e.m. (d) Representative images of cells measured in a before Tg stimulation. Scale bars, $10 \mu \mathrm{m}$.

expression and co-transfected HEKO1 cells with STIM2.1 together with STIM2.2, STIM1 or an equimolar amount of YFP containing a control vector. Co-overexpression of STIM2.2 and the control vector in HEKO1 cells results in an extremely high basal $\mathrm{Ca}^{2+}$, indicating indeed a high activity of coupled STIM2.2 and Orail. Nonetheless, STIM2.2 still elicits additional activation of Orail after store depletion in $0 \mathrm{mM} \mathrm{Ca}^{2+}$ (Fig. 6a-e). Keeping STIM2.2 constant and co-expressing STIM2.1 significantly decreased pre-coupled and full-depletion-operated $\mathrm{Ca}^{2+}$ (Fig. 6a-e). Analysed cells were not sorted for preactivated cells. We also performed whole-cell patch-clamp recordings of HEKO1 cells co-transfected with either splice variant alone and equimolar vector control or with STIM2.2 and STIM2.1. In contrast to the imaging results, co-expression of STIM2.1 did not dampen STIM2.2-mediated activation of Orail in conditions of extreme intracellular $\mathrm{Ca}^{2+}$ buffering (20 mM BAPTA; IP 3 ) (Fig. 6f,h,i). To more closely mimic the $\mathrm{Ca}^{2+}$-imaging condition with only very slightly buffered intracellular $\mathrm{Ca}^{2}+$ (Fura2), we clamped the intracellular free $\mathrm{Ca}^{2+}$ concentration to $150 \mathrm{nM}$ with addition of $\mathrm{IP}_{3}$ and repeated the patch-clamp analysis (Fig. 6g,j,k). Now coexpression showed a significant inhibitory effect in the initial store-operated phase (grey box in Fig. 6g): between 12 and $90 \mathrm{~s}$ after break-in, 32 of 41 time points showed significantly $(P<0.05)$ reduced current densities ( $n=22$ cells for each condition) with co-expression of STIM2.1 and STIM2.2. Expression of STIM2.1 did not elicit currents in either condition. Analyses of maximal current densities and exemplary IV relations are shown in Fig. $6 \mathrm{~h}-\mathrm{j}$. Primary human CD4 ${ }^{+} \mathrm{T}$ cells as well as Jurkat $\mathrm{T}$ cells used in Figs 2 and 3 express both STIM1 and STIM2 (Fig. 1); therefore, we also analysed co-overexpression of STIM2 splice variants with STIM1. Co-expression of STIM2.2 with STIM1 in HEKO1 cells leads to an expected significant increase in basal $\left[\mathrm{Ca}^{2+}\right]_{\mathrm{i}}$ (Supplementary Fig. 3a); however, STIM1-mediated store depletion-induced $\mathrm{Ca}^{2}+$ entry (green trace) was not much affected by co-expression of STIM2.2 analysed within $24 \mathrm{~h}$ after transfection (Supplementary Fig. 3a-e). In contrast, while co-expression of STIM2.1 together with STIM1 did not have a significant effect on basal $\left[\mathrm{Ca}^{2+}\right]_{\mathrm{i}}$, it significantly inhibited SOCE (Supplementary Fig. 3a-e) thus confirming a negative regulatory role of STIM2.1 in conditions of full store depletion as also seen for naive $\mathrm{CD}^{+} \mathrm{T}$ cells and Jurkat $\mathrm{T}$ cells (Figs 2c,g and 3). Whole-cell patch-clamp recordings of HEKS1 cells (stable STIM1 expression), with co-overexpression of Orail together with vector only, STIM2.2 or STIM2.1 revealed that in strong buffering conditions $\left(20 \mathrm{mM}\right.$ BAPTA, $\left.\mathrm{IP}_{3}\right)$ co-expression of both STIM2.2 and STIM2.1 increased STIM1-mediated Orail current densities (Supplementary Fig. 3f-h). Clamping $\left[\mathrm{Ca}^{2+}\right]_{\mathrm{i}}$ to $150 \mathrm{nM}$ in HEKO1 cells upon co-expression of STIM1 with STIM2.1 or STIM2.2 showed smaller currents with very variable kinetics, thus potential inhibitory effects may be difficult to detect (Supplementary Fig. 3i-k). However, both sets of patch-clamp results (Fig. 5; Supplementary Fig. 3) confirm a direct or indirect $\mathrm{Ca}^{2+}$-dependent effect in STIM2.1's ability to act as an inhibitory subunit of SOCE.

Homology modelling and protein-protein docking analysis. To correlate the observed phenotype of STIM2.1 to available structural information on STIM1, we applied homology modelling to predict the structures of STIM2.2 and of STIM2.1 carrying the VAASYLIQ insertion. Homodimers of STIM2.2 and STIM2.1 were modelled according to the structure of the STIM1 dimer (PDB ID: 3TEQ). Details on the applied algorithms and methods as well as some additional validation are given in the Supplementary Material. One certainly needs to acknowledge that, in the case of STIM2.1, the accuracy of modelling such a 
a

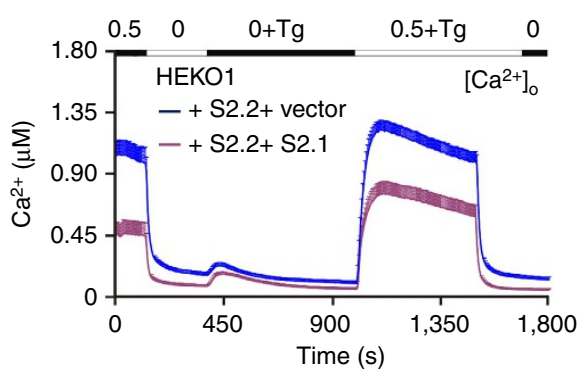

f

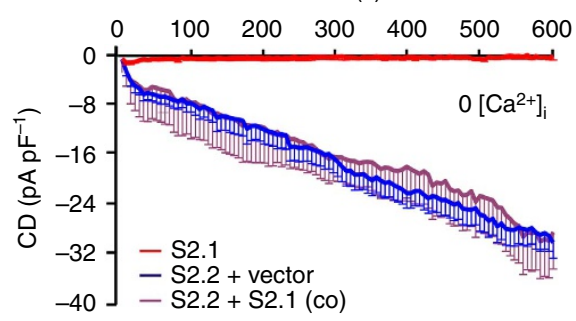

h
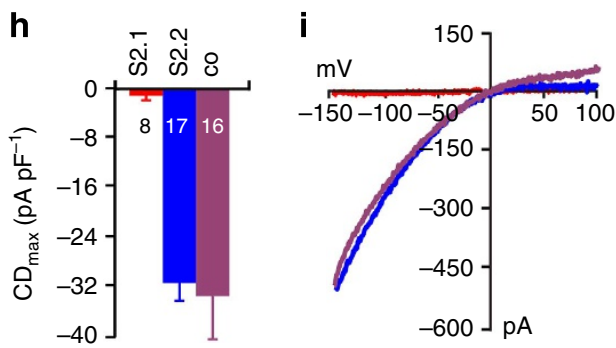

b

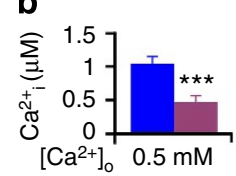

C
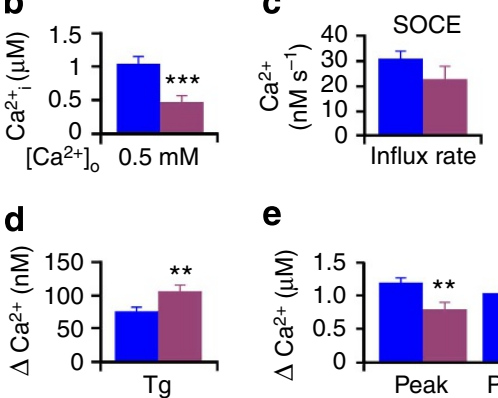

e

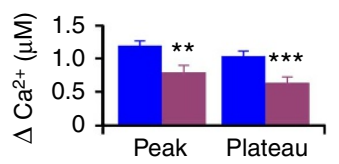

g Time (s)

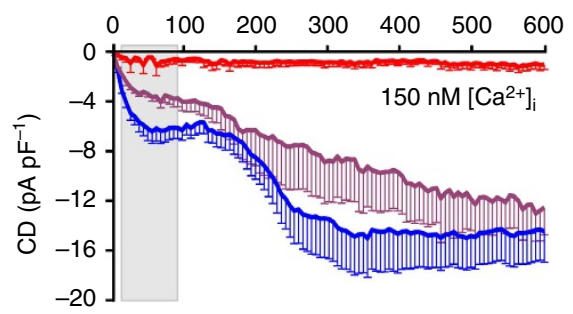

j
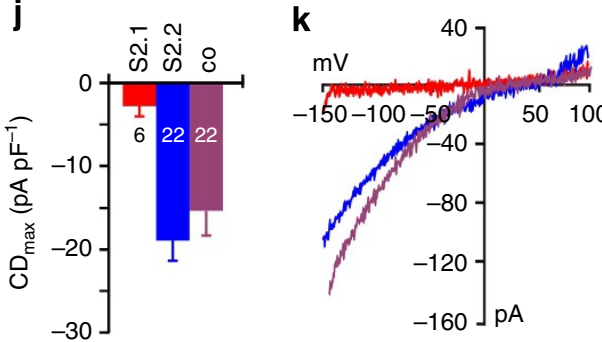

Figure 6 | STIM2.1 opposes STIM2.2 function. (a) Traces showing average changes in intracellular $\mathrm{Ca}^{2}+$ concentration $\left[\mathrm{Ca}^{2+}\right]_{\mathrm{i}}$ over time in response to perfusion of different $\left[\mathrm{Ca}^{2+}\right]_{0}$ indicated in the upper bar in HEKO1 cells co-transfected with $0.5 \mu \mathrm{g}$ CFP-STIM2.2 together with YFP-control vector (blue) or $1 \mu \mathrm{g}$ YFP-STIM2.1 (purple). (b-e) Quantification of changes in $\left[\mathrm{Ca}^{2+}\right]_{i}$ measured in a from 66-69 cells of 6 experiments. (f) Average traces showing wholecell current density (CD) over time extracted at $-130 \mathrm{mV}$ in HEKO1 cells co-transfected like in a or with $1 \mu \mathrm{g}$ YFP-STIM2.1 (red, same trace as Fig. 4f) with internal solution clamping $\left[\mathrm{Ca}^{2+}\right]$ to 0 . (g) Average traces showing whole-cell CD over time measured from cells transfected like in $\mathbf{f}$ with an internal solution clamping $\left[\mathrm{Ca}^{2+}\right]$ to $150 \mathrm{nM}$. Grey box denotes time points between 12 and $90 \mathrm{~s}$ after break-in. (h,j) Average maximum CD recorded from cells measured in $\mathbf{f}$ and $\mathbf{g}$, respectively. (i,k) Current-voltage $(I-V)$ relationship of representative cells recorded at maximal current in $\mathbf{f}$ and $\mathbf{g}$, respectively.

${ }^{\star \star} P<0.01,{ }^{\star \star \star} P<0.001$, Student's $T$-test. Data obtained are presented as mean \pm s.e.m.

large insertion by homology modelling is somewhat limited. Thus, we have considered four alternative cases for STIM2.1 (Fig. 7a; Supplementary Fig. a) where the 8 amino-acid insertion leads either to an ideal extension of helix $S \alpha 1$ (model 1), a flexible loop (model 2) or a partial helix (models 3 and 4). Upon docking these dimer models of the STIM CAD domains to the Orail C-terminal helix using the three different docking packages DOT2 (ref. 27), FRODOCK ${ }^{28}$ and $\mathrm{ZDOCK}^{29}$, this extension turned out to have a significant, consistent impact on the predicted Orail-binding sites. For the STIM1 CAD dimer, all best-scoring solutions generated by the packages predicted the Orail C-terminal helix to bind to motif 1 of STIM1 (Fig. 7b). In contrast, Orail was predicted to favour binding to motifs 2 and 3 of STIM2.2 and of the four STIM2.1 models (Fig. 7b; Supplementary Fig. $4 \mathrm{~b}$ ). Note that due to the symmetric dimer geometry, some configurations docked to the left or right monomer (mostly in motif 3) are in fact (almost) equivalent. On the basis of the proposed model of STIM1-Orail coupling reviewed in ref. 13, the C-terminal helix of Orail can access the upper part of the CAD domain but hardly is able to approach its lower part, which is close to the dimerization domain $(\mathrm{Dd})$ of CAD. Thus, the postulated docking positions in motifs 2 and 3 are likely not biologically active. To rationalize the docking results, we calculated the electrostatic potential around all CAD domains and the Orail C-terminal helix with the software $\mathrm{APBS}^{30}$ and plotted the potential on the corresponding solventaccessible surfaces. As shown in Supplementary Fig. 5, a large fraction of the surface area of Orail helix is negatively charged. To make energetically favourable interactions, the Orail helix should align its negatively charged surface with the positively charged strip on the STIM1 CAD domain. This is likely the reason why the Orail helix was preferably oriented in the upper part of the STIM1 CAD domain termed motif 1 by the three programs (Fig. 7b). Compared with STIM1, the positively charged areas around motif 1 shrink in size in the STIM2.2 and STIM2.1 CAD domains (Fig. 7c; Supplementary Fig. 4c). Besides, in models 2, 3 and 4 of STIM2.1 CAD, the protrusion of the loop (or partial loops) in motif 1 made the interaction surfaces less smooth than the surface of an ideal helix. As a result, alternative positions for the Orail helix (near motifs 2 and 3) were predicted to be energetically more favourable for binding to the STIM2.2 and STIM2.1 models (Fig. 7b; Supplementary Fig. 4b). As argued above, these alternative positions are likely biologically not plausible. Figure 7c shows that the electrostatics of the Dd of STIM2.1 model 1 CAD is quite different from that of STIM1 and STIM2.2. Precisely, the Dds of STIM1 and STIM2.2 CAD are 
a

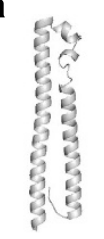

STIM1

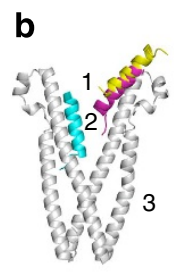

STIM1

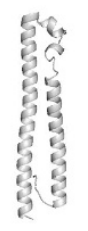

STIM2.2

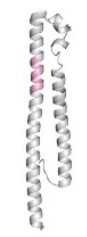

STIM2.1

(Model 1)
C

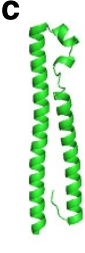

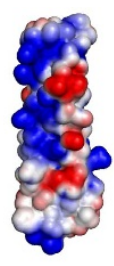

STIM1

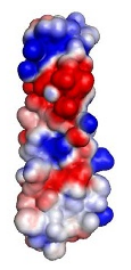

STIM2

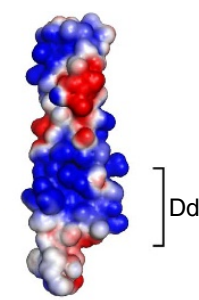

STIM2.1 (model 1)

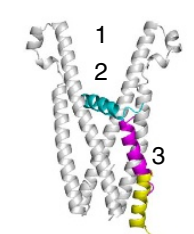

STIM2.2

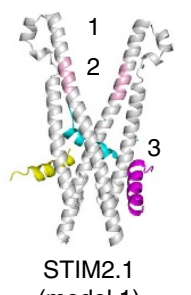

(model 1)
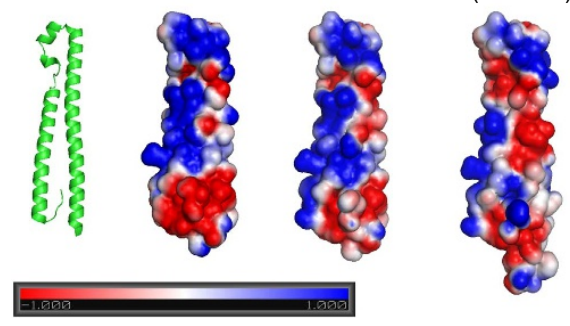

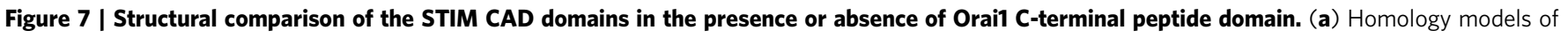

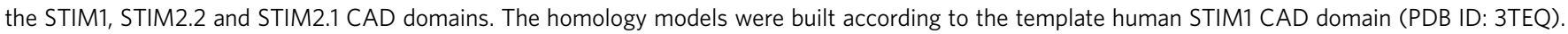

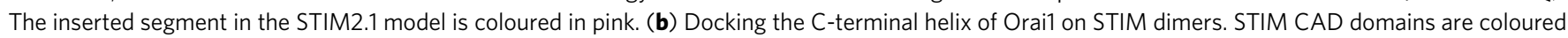
in grey. The conformations of the C-terminal helix of Orai1 were predicted by the docking packages DOT2 (cyan), FRODOCK (magenta) and ZDOCK (yellow). Motif 1: upper part of CAD domain that is supposed to be close to or in contact with the membrane containing ORAI channel proteins. Motif 2: region near the crossing of STIM CAD dimerization domain. Motif 3: the lateral face of STIM CAD near the dimerization domain. (c) Electrostatic potential distribution on the solvent-accessible surfaces of the STIM CAD domains. The unit of electrostatic potential used is kTe ${ }^{-1}$. Dimerization domain (Dd).

relatively neutral, whereas the domains are more positively charged in STIM2.1 model 1. This suggests that STIM2.1 CAD may not function as a stable dimer due to repulsion between its equally charged domains.

Biochemistry of the STIM2.1 and STIM2.2 CAD domains. To obtain further biochemical evidence of splice variant differences and, as the insertion altered the scores of predicted calmodulin (CaM)-binding sites ${ }^{31}$, we tested the amino acids relevant for binding of the Orail C-terminal sequence to both the STIM2.2 and to the STIM2.1 CAD domains by probing immobilized peptide sequences either with ${ }^{14}$ C-labelled glutathione $S$ transferase (GST-CaM) or with a biotinylated Orail C-terminal peptide. Each peptide spot contains a 15-amino-acid sequence starting with Y452 (translated from first ATG (NT 268) of NM001169118.1). The subsequent spot started with a three amino-acid shift generating spots with an overlap of 12 identical amino acids (aa) to the next neighbour (Supplementary Fig. 6). Figure 8 shows images of membranes after phosphoimager development (Fig. 8a) or chemiluminescence detection (Fig. 8b). Analysis of resulting signals shows that biotinylated Orail binds with high specificity to spot 12 (KRSTVFGTLHVAHSS) present in both splice variants (spot 41 is identical, see Supplementary Methods). Both the preceding spot 11 (IKKKRSTVFGTLHVA) and the following spot 13 (TVFGTLHVAHSSSLD) show nearly no binding, indicating that the presence of K477 and R478 as well as of H489 (highlighted in bold in the above sequence of spot 12) is likely critical for Orail binding to STIM2. Binding of CaM shows splice-specific differences: Here inclusion of the 8 aa adds an almost classic CaM-binding motif (IQxxxKIxxxRxxV) present in STIM2.1 spot 9 (LIQAEKIKKKRSTVF). STIM2.2 sequences lack this motif but also bind well to CaM with highest binding to spot 36 (MQLAIAKDEAEKIKK) (Fig. 8c,d). Spots 45-50, derived from a putative in silico CaM-binding site located upstream of the transmembrane domain, display only weak CaM binding. Overlay of both Orail- and CaM-binding motifs on the linear sequence indicates partial overlap of these sites, indicating that binding of either Orail or CaM may be mutually exclusive (Fig. 8d). To further analyse CaM binding, we expressed STIM2.1 and STIM2.2 CAD domains in Escherichia coli, purified and partially refolded the proteins and binding to $\mathrm{CaM}$ with CaMsepharose pull-down assays in buffers containing $\mathrm{Ca}^{2}+$ or EGTA. Supplementary Figure 7 shows that both STIM2 CAD domains bind efficiently to CaM-sepharose in a $\mathrm{Ca}^{2}+$-dependent manner (Supplementary Fig. 7a). With subtraction of background (agarose bound) signal, we uncovered a small difference in binding between the variants (Supplementary Fig. 7b). We performed surface plasmon resonance spectroscopy (SPR) to determine relative affinities for binding of CAD domains to immobilized GST-CaM (Fig. 8e-g). No binding was observed in the absence of $\mathrm{Ca}^{2+}$, confirming results shown in Supplementary Fig. 7. However, in the presence of $\mathrm{Ca}^{2+}$, the STIM2.1 CAD domain shows a significantly higher affinity with a binding constant of $9.1 \pm 2.7 \mathrm{nM}$ compared with $40.7 \pm 10.5 \mathrm{nM}$ for STIM2.2. These results suggest that STIM2.2 and STIM2.1 are affected by both the local $\mathrm{Ca}^{2+}$ and $\mathrm{CaM}$ concentration. However, even with a mutated IQ motif, STIM2.1 is unable to activate Orail (unpublished data).

\section{Discussion}

In our study, we report the identification and functional analysis of a hitherto unknown STIM2 splice variant, STIM2.1, where the sequence VAASYLIQ is spliced into the CAD/SOAR $(\mathrm{S} \alpha 1)$ domain. We show that STIM2.1 is expressed in many cell types, where its relative expression ratio to the known variant STIM2.2 can differ up to eightfold in the cell types we tested. SiRNAmediated downregulation of STIM2.1 in primary naive human $\mathrm{CD} 4{ }^{+}$cells, which express a high amount of both STIM2 splice variants (Fig. 1), shows that thapsigargin-induced SOCE is increased with no effect on basal $\mathrm{Ca}^{2}+$. Given the $44 \%$ remaining relative expression level of STIM2.1 in the siRNA-transfected cells, complete knockout of STIM2.1 is very likely to display an 
a ${ }^{14} \mathrm{C}$ Calmodulin (CAM)

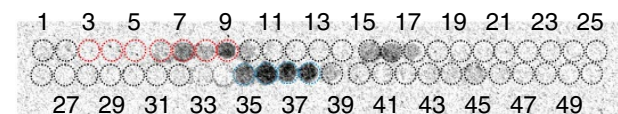

$\begin{array}{llllllllllll}27 & 29 & 31 & 33 & 35 & 37 & 39 & 41 & 43 & 45 & 47 & 49\end{array}$

Spots containing exon 9 sequences STIM2.2/2.1 conserved sequences STIM2.2 exon $8 / 10$ sequences

C $\square$ CaM binding

$\left({ }^{*} 10^{3} \mathrm{RU}\right)$ b

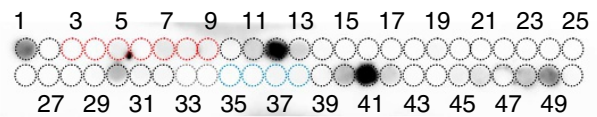
Orai1: Biotin-HKTDRQFQELNELAEFARLQDQLDHR

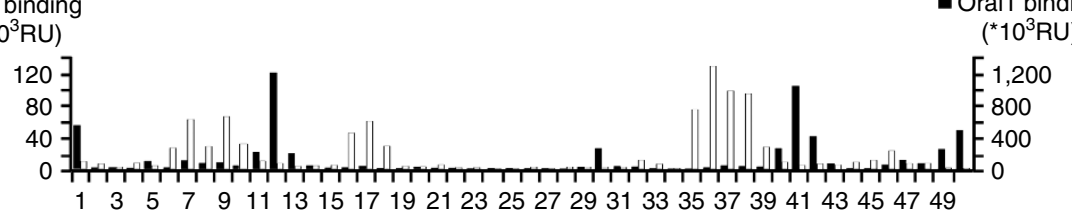

d
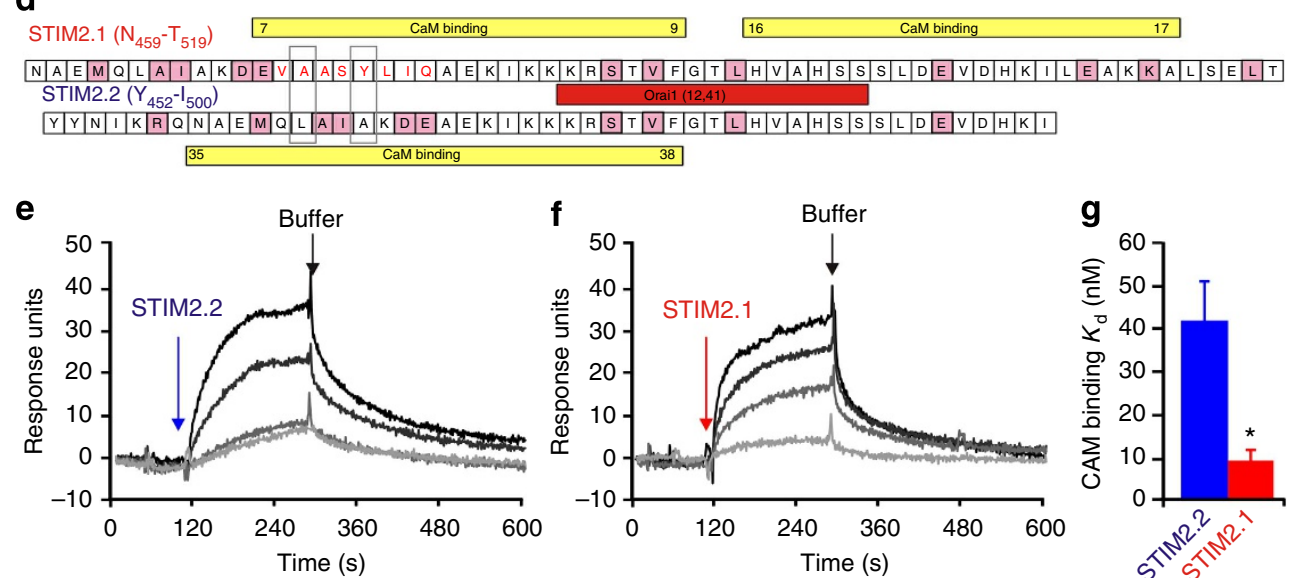

Figure 8 | Characterization of Orai1 and Calmodulin-binding properties of STIM2.1 and STIM2.2. (a) ${ }^{14} \mathrm{C}$-labelled calmodulin (CaM) binding to STIM2 peptide arrays. (b) Biotinylated Orai1 C-term peptide binding to identical STIM2 peptide array as in a. Spots encircled with a red dotted line represent peptides encoding exon 9 sequences, blue dotted line represent peptides encoding the sequence flanking exon 9 (exon 8/10 transition unique in STIM2.2) and black circles represent peptides encoding conserved sequence in both splice variants. (c) Quantification of bound Orai1 peptide (black bars) or CaM (white bars) to the different peptide spots. (d) Schematic representation of partial sequences of STIM2.1 (upper sequence) or STIM2.2 (lower sequence) encoded by the spotted peptides, showing the domains binding to CaM in STIM2.1 (yellow, encoded by peptides 7-9 and 16-17) and in STIM2.2 (yellow, encoded by peptides 35-38) and the identical Orai1-binding domain (red encoded by peptide 12 for STIM2.1 and peptide 41 for STIM2.2). (e,f) Surface plasmon resonance (SPR) analysis of the binding affinity of calmodulin with representative binding curve sensorgrams showing real association and dissociation of purified CAD domains of STIM2.2 (red-filled boxes denote differences to STIM1). (e) or STIM2.1 (f) with immobilized CaM. (g) Average $K_{d}$ values for CaM binding of STIM2.2 (blue) or STIM2.1 (red) obtained from five independent experiments using CAD concentrations ranging from 7.5 to $750 \mathrm{nM},{ }^{*} P<0.05$, Student's T-test. Data obtained are presented as mean \pm s.e.m.

even stronger amplification of SOCE and may uncover a phenotype on basal $\left[\mathrm{Ca}^{2+}\right]_{\mathrm{i}}$. Basal $\mathrm{Ca}^{2+}$ levels increase during T-cell activation ${ }^{32}$ and although it is tempting to speculate that this increase in basal $\left[\mathrm{Ca}^{2+}\right]_{i}$ is due to decreased levels of STIM2.1, expression of both STIM2.2 and STIM2.1 decreases after bead contact and remains low in effector cells, whereas STIM1 expression recovers after $48-72 \mathrm{~h}$ and now is stronger than the sum of both STIM2 splice variants (Fig. 1c). In naive cells, downregulation of STIM2.1 is in contrast to the splicespecific downregulation of STIM2.2, which reduced both basal $\mathrm{Ca}^{2+}$ as well as SOCE (Fig. 2). Database mining ${ }^{33}$ indicates that $\mathrm{CaM}$ is also more highly expressed in naive than in effector $\mathrm{CD}^{+}$cells. Interestingly, overexpression of both variants in Jurkat T cells (Fig. 3) leads to a slight increase in basal $\mathrm{Ca}^{2+}{ }_{\mathrm{i}}$. The effects upon downregulation (Fig. 2) and overexpression (Figs 3 and 6; Supplementary Fig. 2) suggest that STIM2.1 acts as a negative regulator of both STIM1 and STIM2.2 only in full store depletion-mediated activation of Orail. The high expression of STIM2.1 (Fig. 1) in primary $\mathrm{CD}^{+} \mathrm{T}$ cells thus may reduce sensitivity towards stimulatory factors and likely aids in maintaining naive cells in an inert state. The inhibitory effect of co-expression in $\mathrm{Ca}^{2+}$-imaging experiments is not apparent in whole-cell patch-clamp recordings when cells are dialysed with high amounts of BAPTA, although single STIM2.1 expression is still unable to activate Orail in such conditions (Fig. 4), consistent with its lack of interaction seen in FRET experiments (Fig. 5). Homology modelling suggests that STIM2.1 shows altered binding configurations and may not form stable CAD dimers due to electrostatic repulsion of its Dds (Fig. 7). Clamping cytosolic $\mathrm{Ca}^{2}+$ to $150 \mathrm{nM}$ in presence of $\mathrm{IP}_{3}$ unmasked a dominant-negative effect of STIM2.1 on the store-operated phase of STIM2.2-mediated currents and abolished amplification of STIM1 currents (Supplementary Fig. 2i-k). Together, these results suggest that either heterodimers of STIM2.1 with STIM2.2 and possibly with STIM1 can become functional in conditions of extreme buffering (high BAPTA, $0\left[\mathrm{Ca}^{2+}\right]$ ) or that STIM2.1 precludes binding of STIM2.2 or STIM1 to Orai1 subunits by steric hindrance only if cytosolic $\mathrm{Ca}^{2+}$ ions are present, the latter we believe to be more likely. A differential $\mathrm{Ca}^{2+}$ requirement for steric hindrance may explain why STIM2.1's inhibitory effect on basal $\mathrm{Ca}^{2+}$ is not always seen upon overexpression (compare basal $\mathrm{Ca}^{2+}$ of Figs 3 and 4 with 
Fig. 6) and would also indicate a clear dependency on its expression level. Direct binding of $\mathrm{Ca}^{2}+$ to the inhibitory domain of STIM1 has been reported ${ }^{34}$, and local cytosolic $\mathrm{Ca}^{2+}$ elevations are required for STIM1 deoligomerization and termination of store-operated $\mathrm{Ca}^{2+}$ entry ${ }^{35}$, but it is unclear how cytosolic $\mathrm{Ca}^{2+}$ affects STIM2 oligo- or deoligomerization. Parvez et al. ${ }^{10}$ showed that STIM2 shows a biphasic activation of Orail with an early store-dependent phase and a slower and store-independent activation due to loss of $\mathrm{CaM}$ through intracellular dialysis (see also Fig. 4). Our comparison of STIM2.1 and STIM2.2 CAD domain binding to CaM using SPR shows that both domains bind to $\mathrm{Ca}^{2+}{ }_{-} \mathrm{CaM}$ with a much higher affinity than the C-terminal site $(10$ and $40 \mathrm{nM}$ versus $\sim 1 \mu \mathrm{M}^{36,37}$ ). STIM2.1 has an increased affinity and an altered CaM-binding motif likely generated in part by the specific aa sequence (IQ). We also show that Orail-and CaM-binding sites overlap on a linear peptide array, giving biochemical evidence to the hypotheses of Parvez et al. ${ }^{10}$ that CaM binding to STIM 2 can prevent its activation of Orail. However, the fact that STIM2.1 by itself is unable to activate Orail even after long dilution of cytosolic factors in the presence of BAPTA, or upon mutation of its IQ motif (unpublished data) argues against CAM binding and permanently occluding the Orail-binding site. STIM2.1 can still clearly co-localize with Orail upon full store depletion (Fig. 5), but the tight interaction with Orail required for gating is impaired (FRET). The reduced interaction may be caused by the electrostatic destabilization of STIM2.1 dimer formation and of the Orail-binding site predicted by molecular modelling analyses (Fig. 7).

Because basal $\left[\mathrm{Ca}^{2+}\right]_{\mathrm{i}}$ is significantly increased with expression of STIM2.2 in HEKO1 cells independent of the nature of its signal peptide (short STIM1 or long STIM2), it is highly unlikely that this constitutive entry is mediated by non-ER-localized STIM2 as postulated by Graham et al. ${ }^{9}$. Besides the steric and electrostatic implications derived from our molecular modelling approach (Fig. 7), the insertion of VAASYLIQ can also be compared with the effects of mutating amino acids in a similar upstream position relative to the Orail-binding site in the STIM1 CAD domain: a STIM1 A376 (corresponding to STIM2.2 A467 = Y in STIM2.1, see box 1 in Fig. 8d) to K mutant constitutively forms punctae at resting ER $\mathrm{Ca}^{2}+$ with no ability to recruit Orail to these ER-PM $\operatorname{sites}^{38}$. Moreover, a STIM1 L373 (=L464 in STIM2.2, = A in STIM2.1 see box 2 in Fig. 8d) to $S$ mutant fails to couple and activate Orail currents ${ }^{39}$. Insertion of VAASYLIQ thus displays a phenotype that is comparable to certain STIM1 mutants within the $S \alpha 1$ helix of SOAR/CAD. In addition, a very recent report ${ }^{40}$ describes the interaction between $\mathrm{CC} 1$ domain preceding the CAD domain and CAD CC3 domain of STIM1 as a critical regulatory mechanism affecting exposure of the $\mathrm{CAD} \mathrm{CC} 2$ domain, which may also be affected in the case of STIM2.1.

The identification of a STIM2 splice variant antagonizing STIM function resets the stage for analysis of contradictory results regarding the physiological role of STIM2 (that is, in cancer and autoimmunity ${ }^{14}$ ) as all studies involving downregulation or knockout would affect both variants, thus dampening a 'true' STIM2.2-mediated effect.

\footnotetext{
Methods

Cell culture and antibodies. All cells were maintained in a $37^{\circ} \mathrm{C}, 5 \% \mathrm{CO}_{2}$ humidified incubator in corresponding medium, namely, minimum essentia medium for HEK293 WT (HEK) and stably expressing Orail (HEKO1), AMIV for human CD4 + cells and RPMI 1640 for E6.1 Jurkat T (Jurkat) cells. All media were supplemented with $10 \%$ fetal calf serum and $1 \%$ penicillin-streptomycin and HEKO1 were maintained in $1 \mu \mathrm{g} \mathrm{ml}^{-1}$ puromycin. HEK cells were passaged by treatment with trypsin/EDTA. For transfection, the indicated amount of DNA was electroporated into HEK cells and Jurkat cells with Nucleofector II or into CD4+
} cells with $4 \mathrm{D}$ Nucleofector core unit according to the manufacturer's instructions
16-20 h before measurements. Human Orail, Orai2 and STIM1 were subcloned into the EcoRV site of either RFP pMAX, or IRES-RFP-pCAGGS. STIM2.2 was purchased with an N-terminal CFP or YFP tag (Addgene). We used this cDNA (STIM2.2: pEX-CMV-SP-YFP-STIM2) and two complementary primers (see Supplementary Table 1) each encoding part of the exon 9 sequence to insert the desired nucleotides into the exon $8 / 10$ boundary. All constructs were confirmed by sequencing. Antibodies used in this study were anti-His Tag (Cell Signaling, 2366 1:1,000); anti-STIM2 (C-term, Sigma, \#S8572, 1:2,000); anti-Calnexin (Stressgen, \#SPA-865, 1:1,000) and anti- $\gamma$-tubulin (Cell Signaling, \#5886, 1:1,000).

Quantitative real-time PCR. For $\mathrm{qRT}-\mathrm{PCR}$, the indicated cell types were harvested in TRIzol (Life Technologies) and stored at $-80^{\circ} \mathrm{C}$ until RNA was isolated following the manufacturer's instructions. SuperScriptTMII Reverse Transcriptase (Life technologies) was used to generate cDNA and subsequent PCR or qRT-PCR was conducted using the QuantiTect SYBR Green Kit (Qiagen) and a CFX96 RealTime System (Bio-Rad) with the primers listed in Supplementary Table 1. For quantification, expression levels are presented as the normalized quantification cycle $(\mathrm{Cq})$ values of the gene of interest to that of TBP (TATA box-binding protein) using the $\Delta \mathrm{CT}$ method (results were comparable when normalized to RNA polymerase).

Small interfering RNA knockdown. Splice-specific siRNA targeting exon 9 or exon $8 / 10$ boundary were used to specifically knock down endogenous STIM2.1 or STIM2.2, respectively. Using electroporation as mentioned above, $8 \mu \mathrm{l}$ of $40 \mu \mathrm{M}$ stock solution of the indicated siRNA or an equivalent concentration of nontargeting RNA was transfected in parallel for the non-silencing control were transfected into naive CD $4+$ cells. Transfection was repeated on the next day and measurements were conducted 14-18 h later. The sequences of the siRNA are listed below. Knockdown efficiency and off-target effects were tested by qRT-PCR, where the mRNA level was normalized to TBP. Sequences of the used siRNA and qRT-PCR primers are listed in Supplementary Table 1.

Electrophysiology. Recordings were performed at room temperature in the tightseal whole-cell configuration, and linear voltage ramps from -150 to $+150 \mathrm{mV}$ were applied as in ref. 6 . The pipette solution contained the following (in $\mathrm{mM}$ ): 120 Cesium-glutamate, $3 \mathrm{MgCl}_{2}$, 20 Cesium-BAPTA, 10 Hepes and 0.05 IP3 (pH 7.2 with $\mathrm{CsOH}$ ). Where indicated, internal solution contained $150 \mathrm{nM}$ free $\mathrm{Ca}^{2+}$. The external solution contained (in $\mathrm{mM}$ ): $120 \mathrm{NaCl}, 10 \mathrm{TEA}-\mathrm{Cl}, 10 \mathrm{CaCl}_{2}, 2 \mathrm{MgCl}_{2}, 10$ Hepes and glucose $(\mathrm{pH} 7.2$ with $\mathrm{NaOH})$.

Fluorescence-based $\mathbf{C a}^{2}+$ imaging. Human $\mathrm{CD}^{+}{ }^{+}$or Jurkat T cells were loaded in suspension with $1 \mu \mathrm{M}$ Fura 2 -AM at room temperature for $25 \mathrm{~min}$ and seeded on poly-ornithine-coated glass coverslips. HEKO1 cells were loaded at $37^{\circ} \mathrm{C}$ for 25 min with slight rotation on an orbital shaker. All experiments were performed using a self-built perfusion chamber with low volume and high solution exchange rate at room temperature. The external $\mathrm{Ca}^{2}+$ Ringer solution contained (in $\mathrm{mM}$ ): $155 \mathrm{NaCl}, 2 \mathrm{MgCl}_{2}$, 10 glucose, 5 Hepes and $0.5 \mathrm{CaCl}_{2}\left(0.5 \mathrm{Ca}^{2+}\right.$ Ringer $)$ or no $\mathrm{CaCl}_{2}$, but 1 EGTA and $3 \mathrm{MgCl}_{2}$ instead $\left(0 \mathrm{Ca}^{2}+\right.$ Ringer) (pH 7.4 with $\left.\mathrm{NaOH}\right)$ Images were analysed with TILLVision software. The absolute intracellular $\mathrm{Ca}^{2+}$ concentration was estimated from the relation $\left[\mathrm{Ca}^{2+}\right]_{\mathrm{i}}=K^{\star}\left(R-R_{\min }\right) /\left(R_{\max }-R\right)$ where the values of $K, R_{\min }$ and $R_{\max }$ were determined from an in situ calibration of Fura 2-AM in Jurkat T cells as described in ref. 41. Quantification of the trace shows the average basal $\left[\mathrm{Ca}^{2+}\right]$, the maximal Tg-induced peak in $\left[\mathrm{Ca}^{2+}\right]_{\mathrm{i}}$, maximal and plateau $\left[\mathrm{Ca}^{2+}\right]_{\mathrm{i}}$ upon readdition of $0.5 \mathrm{mM}\left[\mathrm{Ca}^{2+}\right]_{\mathrm{o}}$ and influx rate of cells. The minimal $\left[\mathrm{Ca}^{2+}\right]_{\mathrm{i}}$ before addition of $\mathrm{Tg}$ or $0.5 \mathrm{mM}\left[\mathrm{Ca}^{2+}\right]_{\mathrm{o}}$ was subtracted from Tg-peak or maximal $\left[\mathrm{Ca}^{2+}\right]_{\mathrm{i}}$, respectively.

TIRF microscopy. HEK293 were co-transfected with $6 \mu \mathrm{g}$ STIM2.1-mcherry or STIM2.2-mcherry-pIRES and $2 \mu \mathrm{g}$ Orail-GFP-pMAX and seeded on 25 -mm glass coverslips $24 \mathrm{~h}$ before measurement. Stores were depleted by incubation with $1 \mu \mathrm{g}$ $\mathrm{Tg}$ in $0 \mathrm{Ca}^{2+}$ Ringer solution for $15 \mathrm{~min}$. A Leica AM TIRF MC system was used as in ref. 6 . The TIRF focal plane was set to acceptor fluorescence and three sets of images (green fluorescent protein (GFP), FRET and mcherry) were captured: GFP was excited using a 488-nm laser (suppression filter BP 525/50); for mcherry, the laser excitation wavelength was $561 \mathrm{~nm}$ (suppression filter BP 600/40) and for FRET image, a 488-nm laser was used (suppression filter BP 600/40). Image acquisition and analysis were performed with LAS (Leica Application suite) FRET module. Acquisition parameters (laser intensity 40\%, exposure time $100 \mathrm{~ms}$ and penetration depth $200 \mathrm{~nm}$ ) were held constant for all three channels. The apparent FRET efficiency $\left(E_{\mathrm{A}}\right)$ was calculated from background-subtracted images using $E_{\mathrm{A}}(\mathrm{i})=\frac{\mathrm{B}-\mathrm{A} \times \beta-\mathrm{C} \times(\gamma-\alpha \times \beta)}{\mathrm{C} \times(1-\beta \times \delta)}$ described by Van Rheenen ${ }^{42}$, where $\mathrm{A}, \mathrm{B}$ and $\mathrm{C}$ stand for donor-, FRET- and acceptor channel, respectively. Bleed through and crosstalk factors $(\alpha, \beta, \gamma$ and $\delta$ ) were determined individually for every experimental day using single transfected cells. To ensure that FRET was compared for identical acceptor to donor ratios, only FRET values of regions of interest enclosing STIM2 clusters, which were within average acceptor to donor ratios \pm one s.d. of the respective experimental day, were used for final analysis. 
Expression and purification of the CAD domains. The CDNA coding for CAD domain of human STIM2.2 or STIM2.1 were amplified using the above mentioned pEX plasmids with primers including BamHI and $\mathrm{XmaI}$ recognition sites and subcloned into a self-modified pET19b vector creating an N-terminal 6 His tag. Plasmids were expressed in E. coli BL21 Rosetta strain. Overnight cultures were inoculated in $2 \times$ YT medium containing $0.4 \%$ glucose and on the next day diluted $1: 20$, grown to an $\mathrm{OD}_{600}$ of 0.5 before protein production was induced with $1 \mathrm{mM}$ isopropyl-b-D-thiogalactoside for $6 \mathrm{~h}$ at $30^{\circ} \mathrm{C}$. Lysis and protein purification were done as in ref. 43. After purification of the His-tagged domains, excess urea and imidazole were removed using illustra NAP-5 Columns (GE Healthcare), and protein was eluted in buffer containing $500 \mathrm{mM} \mathrm{NaCl}, 50 \mathrm{mM}$ Tris and $0.1 \%$ Triton $\mathrm{X}-100, \mathrm{pH}$ 7. Fractions of the protein were collected and protein concentration was determined using the BCA protein assay kit (Pierce).

Homology modelling and protein-protein docking analyses. Full details of STIM2 homology modelling and protein-protien docking analyses is given in Supplementary Tables 2-4, Supplementary Figs 8 and 9 and Supplementary Methods.

Surface plasmon resonance analysis. SPR spectroscopy was carried out in a BIAlite X system. Briefly, monoclonal goat anti-GST serum (BIACORE, Freiburg, Germany) was immobilized on a CM5 research grade sensor chip (BIACORE) by amine coupling according to the manufacturer's instructions. The chip was equilibrated with application buffer (10 mM HEPES pH 7.4, $150 \mathrm{mM} \mathrm{NaCl}, 6.4 \mathrm{KCl}$, $2 \mathrm{mM} \mathrm{MgCl}_{2}$ and $0.005 \% \mathrm{P} 20$ ) at a flow rate of $20 \mu \mathrm{lmin}^{-1}$. GST-CaM was bound to the immobilized antibodies in the measuring cell. Similarly, immobilized GST in the reference cell served as a negative control. Subsequently, solutions containing increasing concentrations of purified STIM2.1 and STIM2.2 CAD domains (7.5-750 nM) were passed over the chip in the presence of $2 \mathrm{mM} \mathrm{Ca}^{2+}$. Each STIM application was followed by application of running buffer. The analysis was carried out employing the BIA evaluation software version 3.1 (BIACORE).

Peptide-spot-binding assay. Peptides encoding for STIM2 CAD domains were synthesized on acid-hardened cellulose membranes, derivatized with a polyethylene glycol spacer as described ${ }^{44}$. Membranes were equilibrated in binding buffer (150 mM NaCl, $50 \mathrm{mM}$ Tris/ $\mathrm{HCl}$ (pH 7.5), 0.1\% Triton X-100 and $1 \mathrm{mM} \mathrm{CaCl} 2$ or $4 \mathrm{mM}$ EGTA) for $2 \mathrm{~h}$ at $4{ }^{\circ} \mathrm{C} .{ }^{14} \mathrm{C}$-labelled GST-CaM was added and incubated at $4{ }^{\circ} \mathrm{C}$ overnight. The membrane was washed with binding buffer three times for 10 min each, dried at room temperature and subjected to phosphorimaging using a Typhoon-Trio imaging device (GE Healthcare). An equivalent peptide filter was incubated overnight with $10 \mu \mathrm{M}$ biotinylated peptide in TBS with $5 \%$ biotin-free Albumin fraction $\mathrm{V}$ and $2.5 \%$ saccarose after blocking in buffer only for $3 \mathrm{~h}$ at room temperature. Blots were washed and developed after incubation with Avidin-HRP and subsequent chemiluminescence detection.

Calmodulin pull-down assay. The purified STIM2.1 and STIM2.2 CAD domains were incubated at a final concentration of $2.5 \mu \mathrm{M}$ in total volume of $250 \mu \mathrm{l}$ with $25 \mu \mathrm{l}$ prewashed CaM-sepharose beads (GE Healthcare) for $1 \mathrm{~h}$ at $4{ }^{\circ} \mathrm{C}$ with overhead rotation. The binding buffer contained $25 \mathrm{mM}$ Tris, $\mathrm{pH} 7.5,150 \mathrm{mM}$ $\mathrm{NaCl}$ and $1 \mathrm{mM} \mathrm{CaCl} 2$ or $1 \mathrm{mM}$ EGTA. Unbound fraction was removed by washing three times with the corresponding buffer and bound fraction was eluted by boiling at $65^{\circ} \mathrm{C}$ for $15 \mathrm{~min}$ with $2 \times$ Laemmli buffer and finally analysed by electrophoresis. The western blot was probed with anti-His antibody (Cell Signaling) at 1:1,000 dilution. Uncropped versions of al blot and gel images are shown in Supplementary Fig. 10.

\section{References}

1. Demaurex, N. \& Frieden, M. Measurements of the free luminal ER $\mathrm{Ca}(2+)$ concentration with targeted 'cameleon' fluorescent proteins. Cell Calcium 34, 109-119 (2003).

2. Prakriya, M. E. Store-operated calcium channels. Curr. Top. Membr. 71, 1-273 (2013).

3. Soboloff, J. et al. Orail and STIM reconstitute store-operated calcium channel function. J. Biol. Chem. 281, 20661-20665 (2006).

4. Brandman, O., Liou, J., Park, W. S. \& Meyer, T. STIM2 is a feedback regulator that stabilizes basal cytosolic and endoplasmic reticulum $\mathrm{Ca} 2+$ levels. Cell 131, 1327-1339 (2007).

5. Zheng, L., Stathopulos, P. B., Li, G. Y. \& Ikura, M. Biophysical characterization of the EF-hand and SAM domain containing Ca2 + sensory region of STIM1 and STIM2. Biochem. Biophys. Res. Commun. 369, 240-246 (2008).

6. Kilch, T. et al. Mutations of the $\mathrm{Ca} 2+-$ sensing stromal interaction molecule STIM1 regulate $\mathrm{Ca} 2+$ influx by altered oligomerization of STIM1 and by destabilization of the Ca2 + channel Orail. J. Biol. Chem. 288, 1653-1664 (2013).

7. Bandara, S., Malmersjo, S. \& Meyer, T. Regulators of calcium homeostasis identified by inference of kinetic model parameters from live single cells perturbed by siRNA. Sci. Signal. 6, ra56 (2013).
8. Williams, R. T. et al. Identification and characterization of the STIM (stromal interaction molecule) gene family: coding for a novel class of transmembrane proteins. Biochem. J. 357, 673-685 (2001).

9. Graham, S. J., Dziadek, M. A. \& Johnstone, L. S. A cytosolic STIM2 preprotein created by signal peptide inefficiency activates ORAI1 in a store-independent manner. J. Biol. Chem. 286, 16174-16185 (2011).

10. Parvez, S. et al. STIM2 protein mediates distinct store-dependent and store-independent modes of CRAC channel activation. FASEB J. 22, 752-761 (2008).

11. Kar, P., Bakowski, D., Di Capite, J., Nelson, C. \& Parekh, A. B. Different agonists recruit different stromal interaction molecule proteins to support cytoplasmic Ca2 + oscillations and gene expression. Proc. Natl Acad. Sci. USA 109, 6969-6974 (2012).

12. Thiel, M., Lis, A. \& Penner, R. STIM2 drives Ca2 + oscillations through storeoperated Ca2 + entry caused by mild store depletion. J. Physiol. 59, 1433-1445 (2013).

13. Soboloff, J., Rothberg, B. S., Madesh, M. \& Gill, D. L. STIM proteins: dynamic calcium signal transducers. Nature reviews. Mol. Cell Biol. 13, 549-565 (2012).

14. Hoth, M. \& Niemeyer, B. A. The neglected CRAC proteins: Orai2, Orai3, and STIM2. Curr. Top. Membr. 71, 237-271 (2013).

15. Gruszczynska-Biegala, J. \& Kuznicki, J. Native STIM2 and ORAI1 proteins form a calcium-sensitive and thapsigargin-insensitive complex in cortical neurons. J. Neurochem. 126, 727-738 (2013).

16. Berna-Erro, A. et al. STIM2 regulates capacitive $\mathrm{Ca} 2+$ entry in neurons and plays a key role in hypoxic neuronal cell death. Sci. Signal. 2, ra67 (2009).

17. Manji, S. S. et al. STIM1: a novel phosphoprotein located at the cell surface. Biochim. Biophys. Acta 1481, 147-155 (2000).

18. Ruano, Y. et al. Identification of novel candidate target genes in amplicons of Glioblastoma multiforme tumors detected by expression and CGH microarray profiling. Mol. Cancer 5, 39 (2006).

19. McAndrew, D. et al. ORAI1-mediated calcium influx in lactation and in breast cancer. Mol. Cancer Ther. 10, 448-460 (2011).

20. Stanisz, H. et al. Inverse regulation of melanoma growth and migration by Orai1/STIM2-dependent calcium entry. Pigment Cell Melanoma Res. 27, 442-453 (2014).

21. Weidinger, C., Shaw, P. J. \& Feske, S. STIM1 and STIM2-mediated $\mathrm{Ca}(2+)$ influx regulates antitumour immunity by CD8 $(+)$ T cells. EMBO Mol. Med. 5, 1311-1321 (2013).

22. Ma, J., McCarl, C. A., Khalil, S., Luthy, K. \& Feske, S. T-cell-specific deletion of STIM1 and STIM2 protects mice from EAE by impairing the effector functions of Th1 and Th17 cells. Eur. J. Immunol. 40, 3028-3042 (2010).

23. Oh-Hora, M. et al. Dual functions for the endoplasmic reticulum calcium sensors STIM1 and STIM2 in T cell activation and tolerance. Nat. Immunol. 9, 432-443 (2008).

24. Cheng, K. T. et al. STIM1 and STIM2 protein deficiency in T lymphocytes underlies development of the exocrine gland autoimmune disease, Sjogren's syndrome. Proc. Natl Acad. Sci. USA 109, 14544-14549 (2012).

25. Wang, X. et al. Distinct Orai-coupling domains in STIM1 and STIM2 define the Orai-activating site. Nat. Commun. 5, 3183 (2014).

26. Schindelin, J. et al. Fiji: an open-source platform for biological-image analysis Nat. Methods 9, 676-682 (2012).

27. Roberts, V. A., Thompson, E. E., Pique, M. E., Perez, M. S. \& Ten Eyck, L. F. DOT2: macromolecular docking with improved biophysical models. J. Comput. Chem. 34, 1743-1758 (2013).

28. Garzon, J. I. et al. FRODOCK: a new approach for fast rotational proteinprotein docking. Bioinformatics. 25, 2544-2551 (2009).

29. Pierce, B. G., Hourai, Y. \& Weng, Z. Accelerating protein docking in ZDOCK using an advanced 3D convolution library. PLoS ONE 6, e24657 (2011).

30. Baker, N. A., Sept, D., Joseph, S., Holst, M. J. \& McCammon, J. A. Electrostatics of nanosystems: application to microtubules and the ribosome. Proc. Natl Acad. Sci. USA 98, 10037-10041 (2001).

31. Yap, K. L. et al. Calmodulin target database. J. Struct. Funct. Genomics 1, 8-14 (2000).

32. Wei, S. H. et al. $\mathrm{Ca} 2+$ signals in $\mathrm{CD} 4+\mathrm{T}$ cells during early contacts with antigen-bearing dendritic cells in lymph node. J. Immunol. 179, 1586-1594 (2007).

33. Wu, C. et al. BioGPS: an extensible and customizable portal for querying and organizing gene annotation resources. Genome Biol. 10, R130 (2009).

34. Mullins, F. M., Park, C. Y., Dolmetsch, R. E. \& Lewis, R. S. STIM1 and calmodulin interact with Orail to induce $\mathrm{Ca} 2+$-dependent inactivation of CRAC channels. Proc. Natl Acad. Sci. USA 106, 15495-15500 (2009).

35. Shen, W. W., Frieden, M. \& Demaurex, N. Local cytosolic Ca2 + elevations are required for stromal interaction molecule 1 (STIM1) de-oligomerization and termination of store-operated Ca2 + entry. J. Biol. Chem. 286, 36448-36459 (2011).

36. Bauer, M. C., O'Connell, D., Cahill, D. J. \& Linse, S. Calmodulin binding to the polybasic C-termini of STIM proteins involved in store-operated calcium entry. Biochemistry 47, 6089-6091 (2008). 
37. Bhardwaj, R., Muller, H. M., Nickel, W. \& Seedorf, M. Oligomerization and $\mathrm{Ca} 2+/$ calmodulin control binding of the ER Ca2 +-sensors STIM1 and STIM2 to plasma membrane lipids. Biosci. Rep. 33, e00077 (2013).

38. Covington, E. D., Wu, M. M. \& Lewis, R. S. Essential role for the CRAC activation domain in store-dependent oligomerization of STIM1. Mol. Biol. Cell 21, 1897-1907 (2010)

39. Frischauf, I. et al. Molecular determinants of the coupling between STIM1 and Orai channels: differential activation of Orail-3 channels by a STIM1 coiledcoil mutant. J. Biol. Chem. 284, 21696-21706 (2009).

40. Fahrner, M. et al. A coiled-coil clamp controls both conformation and clustering of stromal interaction molecule 1 (STIM1). J. Biol. Chem. 289, 33231-33244 (2014)

41. Grynkiewicz, G., Poenie, M. \& Tsien, R. Y. A new generation of Ca2 + indicators with greatly improved fluorescence properties. J. Biol. Chem. 260, 3440-3450 (1985).

42. van Rheenen, J., Langeslag, M. \& Jalink, K. Correcting confocal acquisition to optimize imaging of fluorescence resonance energy transfer by sensitized emission. Biophys. J. 86, 2517-2529 (2004).

43. Al-Ansary, D., Bogeski, I., Disteldorf, B. M., Becherer, U. \& Niemeyer, B. A. ATP modulates Ca2 + uptake by TRPV6 and is counteracted by isoformspecific phosphorylation. FASEB J. 24, 425-435 (2010).

44. Hilpert, K., Winkler, D. F. \& Hancock, R. E. Peptide arrays on cellulose support: SPOT synthesis, a time and cost efficient method for synthesis of large numbers of peptides in a parallel and addressable fashion. Nat. Protoc. 2, 1333-1349 (2007).

\section{Acknowledgements}

We thank the Institute for Clinical Hemostaseology and Transfusion Medicine for providing donor blood. We thank Drs Schwarz, Ludwig and Meese for supplying donor cDNA, A. Knörck and SFB894 projects P1 and P3 (DFG) for technical support, Drs Hoth and Peinelt for equipment support, constructs, helpful discussions and critical reading of the manuscript. D.A. acknowledges funding support from HOMFORExcellent (UdS). P.H.L. and V.H. were supported by SFB1027 (C3). B.A.N. was supported by grant SFB894 (A2) and SFB1027 (C4) from the Deutsche Forschungsgesellschaft (DFG). A.-M.M. is a member of the IRTG1830. M.J. was supported by SFB894 (P3).

\section{Author contributions}

A.-M.M., D.A. and B.A.N. conceived the project, designed the experiments and wrote the manuscript. A.-M.M. and D.A. performed the experiments and analysed the data. G.S. contributed the qRT-PCR analysis and gave technical support. P.-H.L. and V.H. undertook homology modelling and protein-protein docking analyses. M.J. designed peptide blots and performed radioactive experiments.

\section{Additional information}

Supplementary Information accompanies this paper at http://www.nature.com/ naturecommunications

Competing financial interests: The authors declare no competing financial interests.

Reprints and permission information is available online at http://npg.nature.com/ reprintsandpermissions/

How to cite this article: Miederer, A.-M. et al. A STIM2 splice variant negatively regulates store-operated calcium entry. Nat. Commun. 6:6899 doi: 10.1038/ncomms7899 (2015).

(c) (i) This work is licensed under a Creative Commons Attribution 4.0 International License. The images or other third party material in this article are included in the article's Creative Commons license, unless indicated otherwise in the credit line; if the material is not included under the Creative Commons license, users will need to obtain permission from the license holder to reproduce the material. To view a copy of this license, visit http://creativecommons.org/licenses/by/4.0/ 